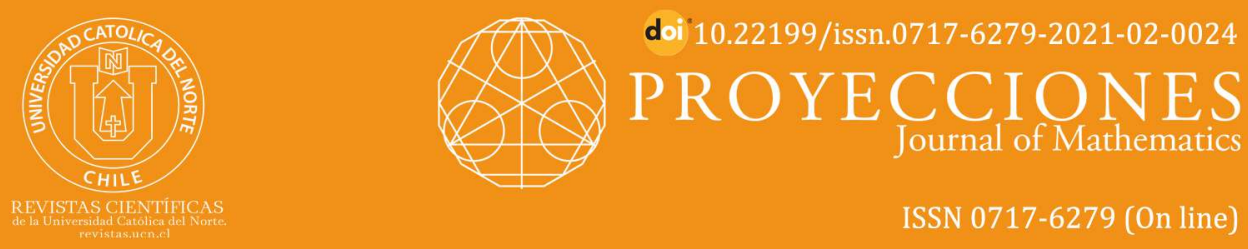

\title{
Some remarks on fuzzy infi topological spaces
}

\author{
Birojit Das ${ }^{1^{*}}$ (0) orcid.org/0000-0001-7614-1051 \\ Baby Bhattacharya ${ }^{2}$ (0) orcid.org/0000-0001-6053-6067 \\ Apu Kumar Saha ${ }^{3}$ (c) orcid.org/0000-0002-3475-018X
}

National Institute of Technology, Dept. of Mathematics, Agartala, TR, India.

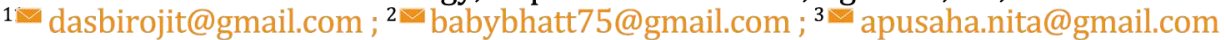

\section{Abstract:}

Induced fuzzy infi topological space is already introduced by Saha and Bhattacharya [Saha A.K., Bhattacharya D. 2015, Normal Induced Fuzzy Topological Spaces, Italian Journal of Pure and Applied Mathematics, 34, 45-56]. In this paper for the said space, we further analyse some properties viz. fuzzy Icontinuity, fuzzy infi open mappings and fuzzy infi closed mappings etc. Also we study product fuzzy infi topological space and establish some results concerned with it.

Keywords: Fuzzy infi topological space; Fuzzy I-continuity; Fuzzy infi open mappings; Fuzzy infi closed mappings; Product fuzzy infi topology.

MSC (2020): 54A40.

Cite this article as (IEEE citation style):

B. Das, B. Bhattacharya, and A. K. Saha, "Some remarks on fuzzy infi topological spaces", Proyecciones (Antofagasta, On line), vol. 40, no. 2, pp. 399-415, 2021, doi: 10.22199/issn.0717-6279-2021-02-0024

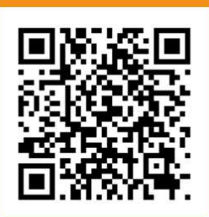

Article copyright: (C) 2021 Birojit Das, Baby Bhattacharya and Apu Kumar Saha. This is an open access article distributed under the terms of the Creative Commons License, which permits unrestricted use and distribution provided the original author and source are credited.

${ }^{*}$ Corresponding author 


\section{Introduction}

The notion of a fuzzy set and fuzzy set operations as an extension of the classical notion of set, were introduced by Zadeh [18] in 1965. The potential has caused great interest among both pure and applied mathematicians. General topology was one of the branches of pure mathematics to which fuzzy sets has been applied systematically. It was in 1968, Chang [5] made the first "grafting" of the notion of a fuzzy set into general topology. Many authors used Chang's definition in many directions to obtain some results which are compatible with results in general topology. In 1976, Lowen [8] suggested an alternative and more natural definition for achieving more results which are compatible to the general case in topology. Since then, many researchers have tried successfully to generalize several pivot concepts of general topology in fuzzy setting $[15,16,17]$. In 1985, Sostak [14] introduced the smooth fuzzy topology as an extension of Chang's fuzzy topology. In 1987, Monsef and Ramadan [11] introduced supra topology in fuzzy senses and named it fuzzy supra topology. These are the natural generalizations corresponding to the notions in general topology as given by Mashhour et al. [10]. In 1994, Dang, Behera, Nanda [7] further investigated and characterized some more properties of supra $\alpha$-compactness and supra $\alpha^{*}$-compactness in fuzzy supra topological space. The concept of minimal structure and minimal spaces, as a generalization of topology and topological spaces were introduced by Maki et. al [9]. Alimohammady and Roohi $[1,2]$ introduced and studied the notions of fuzzy minimal structures and fuzzy minimal spaces. Moreover, Alimohammady et. al [3] also discussed the separation axiom in fuzzy minimal spaces. Chetty [12], introduced the concept of generalized fuzzy topology. This is also a generalization of fuzzy topological spaces. In [6], authors introduced infi topological spaces as a generalization of topological spaces and studied various properties of that space. Saha and Bhattacharya [13] introduced and characterized the concept of fuzzy infi topological spaces, which is also a generalization of fuzzy topological spaces, inducing with NLSC functions.

In this paper we further analysed the fuzzy infi topological spaces. We studied the basic properties of fuzzy topological space like fuzzy interior, fuzzy closure, continuity, fuzzy open mapping and fuzzy closed mapping, product fuzzy topology etc in the fuzzy infi topological spaces. 


\section{Preliminaries}

Before proceeding to the main results we need some preliminary ideas of fuzzy topology, fuzzy supra topology, fuzzy minimal space etc.

\subsection{Definition [5]}

A family $\tau \subseteq I^{X}$, where $I^{X}$ is the collection of all functions from $X$ to the interval $I=[0,1]$, of fuzzy sets in $X$ is called a fuzzy topology if it satisfies the following conditions:

(i) $\overline{0}, \overline{1} \in \tau$,

(ii) if $\lambda$ and $\mu \in \tau$, then $\lambda \wedge \mu \in \tau$,

(iii) if $\lambda_{i} \in \tau$, for each $i=1,2,3, \ldots$, then $\vee \lambda_{i} \in \tau$.

$\tau$ is called a fuzzy topology for $X$, and the pair $(X, \tau)$ is a fuzzy topological space, or fts for short. Every member of $\tau$ is called a $\tau$-open fuzzy set. A fuzzy set is $\tau$-closed if and only if its complement is $\tau$-open.

\subsection{Definition [4]}

Let $(X, \tau)$ and $(Y, \sigma)$ be two fuzzy topological spaces and $f: X \rightarrow Y$ be a function. $f$ is called fuzzy continuous if the inverse image under $f$ of any open fuzzy set in $Y$ is an open fuzzy set in $X$; i.e., if $f^{-1}(v) \in \tau$, whenever $v \in \sigma$.

\subsection{Definition [9]}

Let $X$ be a set. A fuzzy supra topology $\tau^{*}$ on $X$ is a collection of subsets of $X$, each called a supra-open set, such that

(i) $\Phi$ and $X$ are open sets,

(ii) The union of any collection of open sets is an open set.

The set $\mathrm{X}$ together with a supra topology $\tau^{*}$ on $X$ is called a supra topological space.

\subsection{Definition [2]}

We say a family $M$ of fuzzy sets in $X$ is a fuzzy minimal structure on $X$ if $\alpha 1_{X} \in M$, for any $\alpha \in I$. We call the structure, $(X, M)$ a fuzzy minimal space. 


\subsection{Definition [6]}

Let $X$ be a non-empty set and $\tau$ be a collection of subsets of $X$ with the following properties

(i) $\Phi, X \in \tau$,

(ii) $\tau$ is closed under finite intersection.

Then $\tau$ is said to be an infi topology on $X$ and the structure $(X, \tau)$ is known as an infi topological space.

\section{Fuzzy Infi Topological Spaces}

Having these preliminary ideas we now analyse fuzzy infi topological space. In this section we first give the definition and existence of a fuzzy infi topological space, as it was given in [13]. Then, we study some important properties of this space. Also we define fuzzy $I$-continuity and establish some results. We also study fuzzy infi open mappings and fuzzy infi closed mappings and investigate their behaviour in the same environment.

\subsection{Definition [13]}

A family $\mathcal{F}$ of fuzzy sets in $X$ is said to form a fuzzy infi topology in $X$ if

(i) $r 1_{X} \in \mathcal{F}$, for $r \in I$,

(ii) for any two fuzzy subsets $\lambda$ and $\mu$ of $\mathcal{F}, \lambda \wedge \mu \in \mathcal{F}$.

The space $(X, \mathcal{F})$ is called fuzzy infi topological space. Every member of $\mathcal{F}$ is called fuzzy infi open set. A fuzzy set $\lambda$ is fuzzy infi closed if its complement is fuzzy infi open.

\subsection{Definition $[13]$}

A function $f: X \rightarrow R$ is called lower semi-continuous if for each $r \in R$, the set $\{x: f(x)>r\}$ is open in $X$, i.e., $\{x: f(x) \leq r\}$ is closed in $X$.

A lower semi-continuous (LSC) function $\phi$ on $X$ is normal (NLSC) iff for each $x \in X, \phi(x)<\lambda$ and an open set $U$ containing $x$, there exists a non-void open set $V$ such that $\bar{V} \subset U$ with $\phi(v)<\lambda$, for each $v \in V$. 


\subsection{Example}

Let us consider the family $\mathcal{F}=\{f \alpha: \alpha \in \Lambda\}$ of all NLSC functions from a topological space $X$ to the closed unit interval $I$. Thus by Theorems 2.10 and 2.12, in [13], we see that $\mathcal{F}$ satisfies the following properties:

(i) $t 1_{X} \in \mathcal{F}$, for all $t \in I$,

(ii) $f_{i} \in \mathcal{F}$, implies infi $i_{i} f_{i} \in \mathcal{F}$.

Thus by Definition 3.1, the family $\mathcal{F}$ of NLSC functions from $X$ to $I$ (henceforth, denoted by $n L(X)$ ) forms a fuzzy infi topological space.

\subsection{Theorem}

Finite union of fuzzy infi closed set in a fuzzy infi topological space is a fuzzy infi closed set.

Proof: It is obvious.

\subsection{Remark}

Intersection of two fuzzy infi closed set is not fuzzy infi closed, in general. It is clear from the following example.

\subsection{Example}

Let $X=\{a, b, c\}$ and $\tau^{*}=\left\{0_{X}, 1_{X},\left\{\left(a, \frac{1}{2}\right),\left(b, \frac{1}{3}\right),\left(c, \frac{1}{4}\right)\right\},\left\{\left(a, \frac{1}{2}\right),\left(b, \frac{1}{4}\right),\left(c, \frac{1}{3}\right)\right\}\right.$, $\left.\left\{\left(a, \frac{1}{2}\right),\left(b, \frac{1}{4}\right),\left(c, \frac{1}{4}\right)\right\}\right\}$ be an fuzzy infi topology defined over it. Now the class of all infi-closed sets is $=\left\{0_{X}, 1_{X},\left\{\left(a, \frac{1}{2}\right),\left(b, \frac{2}{3}\right),\left(c, \frac{3}{4}\right)\right\},\left\{\left(a, \frac{1}{2}\right),\left(b, \frac{3}{4}\right),\left(c, \frac{2}{3}\right)\right\}\right.$, $\left.\left\{\left(a, \frac{1}{2}\right),\left(b, \frac{3}{4}\right),\left(c, \frac{3}{4}\right)\right\}\right\}$. Let us consider two fuzzy infi closed sets $\lambda$ and $\mu$ such that $\lambda=\left\{\left(a, \frac{1}{2}\right),\left(b, \frac{2}{3}\right),\left(c, \frac{3}{4}\right)\right\}$ and $\mu=\left\{\left(a, \frac{1}{2}\right),\left(b, \frac{3}{4}\right),\left(c, \frac{2}{3}\right)\right\}$. Then $\lambda \wedge \mu=\left\{\left(a, \frac{1}{2}\right),\left(b, \frac{2}{3}\right),\left(c, \frac{2}{3}\right)\right\}$. Thus $\lambda \wedge \mu$ is not fuzzy infi closed.

\subsection{Definition}

For a fuzzy set $\lambda$ in fuzzy infi topological space $X$, the fuzzy infi interior $\lambda_{i i}$ of $\lambda$ is the union of all fuzzy infi open sets contained in $\lambda$, and the fuzzy infi closure $\lambda_{i c}$ of $\lambda$ is the intersection of all fuzzy infi closed sets containing $\lambda$. 


\subsection{Theorem}

If $\lambda$ and $\mu$ are two fuzzy sets in the fuzzy infi topological space $X$, then

- $\lambda_{i i} \leq \lambda$ and $\lambda$ is fuzzy infi open iff $\lambda=\lambda_{i i}$

- $\mu \leq \mu_{i c}$ and $\mu$ is fuzzy infi closed iff $\mu=\mu_{i c}$

- If $\lambda \leq \mu$, then $\lambda_{i i} \leq \mu_{i i}$ and $\lambda_{i c} \leq \mu_{i c}$

- $(\lambda \wedge \mu)_{i i}=\lambda_{i i} \wedge \mu_{i i}$ and $\lambda_{i i} \vee \mu_{i i} \leq(\lambda \vee \mu)_{i i}$

- $(\lambda \vee \mu)_{i c}=\lambda_{i c} \vee \mu_{i c}$ and $(\lambda \wedge \mu)_{i c} \leq \lambda_{i c} \wedge \mu_{i c}$

- $\left(\lambda_{i i}\right)_{i i}=\lambda_{i i}$ and $\left(\lambda_{i c}\right)_{i c}=\lambda_{i c}$

- $\left(\lambda_{i c}\right)^{c}=\left(\lambda^{c}\right)_{i i}$ and $\left(\lambda_{i i}\right)^{c}=\left(\lambda^{c}\right)_{i c}$

Proof: The proofs are obvious.

\subsection{Definition}

Let $(X, \tau)$ be a fuzzy topological space and $\tau^{*}$ be a fuzzy infi-topology on $X$. We call $\tau^{*}$ a fuzzy infi-topology associated with $\tau$ if $\tau \subset \tau^{*}$.

\subsection{Definition}

Let $\left(X, \tau_{1}\right)$ and $\left(Y, \tau_{2}\right)$ be fuzzy topological spaces and $\tau_{1}^{*}$ and $\tau_{2}^{*}$ be associated fuzzy infi-topologies with $\tau_{1}$ and $\tau_{2}$ respectively. A function $f: X \rightarrow Y$ is a fuzzy I-continuous function if the inverse image of each $\tau_{2}^{*}$ fuzzy infi open set in $Y$ is $\tau_{1}^{*}$-fuzzy infi open in $X$. That is, a mapping $f:\left(X, \tau_{1}^{*}\right) \rightarrow\left(Y, \tau_{2}^{*}\right)$ between two fuzzy infi topological spaces is called fuzzy infi continuous or fuzzy I-continuous function if $f^{-1}\left(\tau_{2}^{*}\right) \subset \tau_{1}^{*}$.

\subsection{Theorem}

Let $\left(X, \tau_{1}^{*}\right)$ and $\left(Y, \tau_{2}^{*}\right)$ be two fuzzy infi topological spaces. Let $f$ be a function from $X$ into $Y$. Then the followings are equivalent:

(i) $f$ is fuzzy I-continuous;

(ii) the inverse image of each fuzzy infi closed set in $Y$ is $\tau_{1}^{*}$-fuzzy infi closed; 
(iii) $\left(f^{-1}(\mu)\right)_{i c} \leq f^{-1}\left(\mu_{i c}\right)$, for all fuzzy set $\mu$ of $Y$;

(iv) $f\left(\lambda_{i c}\right) \leq\left(f(\lambda)_{i c}\right.$, for all fuzzy set $\lambda$ of $X$;

(v) $f^{-1}\left(\mu_{i i}\right) \leq\left(f^{-1}(\mu)\right)_{i i}$, for every fuzzy set $\mu$ in $Y$;

(vi) for each fuzzy set $\lambda$ in $X$ and each fuzzy neighborhood $\eta$ of $f(\lambda)$, there is a fuzzy infi neighborhood $\beta$ of $\lambda$ such that $f(\beta) \leq \eta$.

Proof: $(i) \Rightarrow(i i)$ : Let $\mu$ be fuzzy infi closed set in $Y$. Then $1-\mu$ is fuzzy infi open in $Y$. Since $f$ is fuzzy I-continuous, $f^{-1}(1-\mu)=1-f^{-1}(\mu)$ is fuzzy infi open in $X$. Therefore $f^{-1}(\mu)$ is a fuzzy infi closed set in $X$.

$(i i) \Rightarrow(i i i)$ : Since $\mu_{i c}$ is fuzzy infi closed for every fuzzy set $\mu$ in $Y, f^{-1}\left(\mu_{i c}\right)$ is $\tau_{1}^{*}$-fuzzy infi closed.

$$
\left(f^{-1}(\mu)\right)_{i c} \leq\left(f^{-1}\left(\mu_{i c}\right)\right)_{i c}=f^{-1}\left(\mu_{i c}\right) .
$$

$($ iii $) \Rightarrow($ iv $)$ : Let $\lambda$ be fuzzy subset in $X$ and let $f(\lambda)=\mu$. Then $\left(f^{-1}(\mu)\right)_{i c} \leq f^{-1}\left(\mu_{i c}\right)$.

So

$$
\lambda_{i c} \leq f^{-1}(f(\lambda))_{i c} \leq f^{-1} \overline{(f(\lambda))}
$$

and hence $f\left(\lambda_{i c}\right) \leq f\left(f^{-1}\left(f(\lambda)_{i c}\right)\right) \leq(f(\lambda))_{i c}$.

$(i v) \Rightarrow(i i)$ : Let $\mu$ be a fuzzy infi closed set in $Y$ and $\lambda=f^{-1}(\mu)$. Then $f\left(\lambda_{i c}\right) \leq(f(\lambda))_{i c}=\left(f\left(f^{-1}(\mu)\right)\right)_{i c} \leq \mu_{i c}=\mu$.

Thus, $\lambda_{i c} \leq f^{-1}\left(f\left(\lambda_{i c}\right) \leq f^{-1}(\mu)=\lambda\right.$. Then $\lambda$ is $\tau_{1}^{*}$ - fuzzy infi closed.

$($ ii $) \Rightarrow(i)$ : Let $\mu$ be a fuzzy infi open set in $Y$. Then $(1-\mu)$ is fuzzy infi closed. Thus, $f^{-1}(1-\mu)=1-f^{-1}(\mu)$ is fuzzy infi-closed in $X$ and hence $f^{-1}(\mu)$ is fuzzy infi-open in $X$. Thus $f$ is fuzzy I-continuous.

$(i) \Rightarrow(v)$ : Let $\mu$ be a fuzzy subset in $Y$. Since $f^{-1}\left(\mu_{i i}\right)$ is $\tau_{1}^{*}$-fuzzy infi open in $X$,

$$
f^{-1}\left(\mu_{i i}\right) \leq\left(f^{-1}\left(\mu_{i i}\right)\right)_{i i} \leq\left(f^{-1}(\mu)\right)_{i i} .
$$

Thus, $f^{-1}\left(\mu_{i i}\right) \leq\left(f^{-1}(\mu)\right)_{i i}$.

$(v) \Rightarrow(i)$ : Let $\mu$ be a fuzzy infi open set in $Y$. Since $f^{-1}\left(\mu_{i i}\right)=f^{-1}(\mu) \leq$ $\left(f^{-1}(\mu)\right)_{i i}$, therefore, $f^{-1}(\mu)$ is $\tau_{1}^{*}$ - fuzzy infi open.

$(i) \Rightarrow(v i)$ : Let $f: X \rightarrow Y$ be fuzzy I-continuous. For any fuzzy set $\lambda$ in $X$ and for any fuzzy open set $\eta$ of $Y$ containing $f(\lambda)$, let $\beta=f^{-1}(\eta)$. Clearly $\beta$ is a neighbourhood of $\lambda$. Now $f(\beta)=f\left(f^{-1}(\eta) \leq \eta\right.$. 
$(v i) \Rightarrow(i)$ : Let $\eta$ be any fuzzy open set in $Y$ and $f^{-1}(\eta)=\beta$. Then $\eta$ is a fuzzy neighbourhood of $f(\beta)=f\left(f^{-1}(\eta)\right)$. There exists a fuzzy infi neighbourhood $\gamma$ of $\beta=f^{-1}(\eta)$ such that $f(\gamma) \leq \beta$. Thus $\gamma \leq f^{-1} f(\gamma) \leq$ $f^{-1}(\eta)$. Therefore, $f^{-1}(\eta)$ is a fuzzy infi neighbourhood of $f^{-1}(\eta)$ and $f^{-1}(\eta)$ is a fuzzy infi open set in $X$.

\subsection{Theorem}

Let $\left(X, \tau_{1}\right),\left(Y, \tau_{2}\right)$ be fuzzy topological spaces and $\tau_{1}^{*}, \tau_{2}^{*}$ be the associated fuzzy infi topologies with $\tau_{1}, \tau_{2}$ respectively. If $f:\left(X, \tau_{1}\right) \rightarrow\left(Y, \tau_{2}\right)$ is a fuzzy mapping, and one of the following:

(i) $f^{-1}\left(\mu_{i i}\right) \leq\left(f^{-1}(\mu)\right)^{0}$, for each fuzzy set $\mu$ in $\left(Y, \tau_{2}\right)$;

(ii) $\overline{\left(f^{-1}(\mu)\right)} \leq f^{-1}\left(\mu_{i c}\right)$, for each fuzzy set $\mu$ in $\left(Y, \tau_{2}\right)$;

(iii) $f(\bar{\lambda}) \leq(f(\lambda))_{i c}$, for each fuzzy set $\lambda$ in $\left(X, \tau_{1}\right)$,

holds, then $f$ is fuzzy continuous.

Proof: Let the condition $(i i)$ is satisfied. Suppose $\mu$ is a fuzzy closed set in $Y$, then $\overline{\left(f^{-1}(\mu)\right)} \leq f^{-1}\left(\mu_{i c}\right)=f^{-1}(\mu)$. Therefore $f^{-1}(\mu)$ is fuzzy closed in $X$.

If the condition (iii) is satisfied, then for any fuzzy set $\mu$ in $Y, f^{-1}(\mu)$ is a fuzzy set in $X$. Then, $f\left(\overline{\left(f^{-1}(\mu)\right)}\right) \leq\left(f\left(f^{-1}(\mu)\right)\right)_{i c}$ and thus $\overline{\left(f^{-1}(\mu)\right)} \leq$ $f^{-1}\left(\lambda_{i c}\right)$. Therefore, as condition (ii) is satisfied, $f$ is fuzzy continuous.

Similarly we can prove the case (i).

\subsection{Theorem}

Every fuzzy continuous mapping is fuzzy I-continuous.

Proof: Let a fuzzy mapping $f:\left(X, \tau_{1}\right) \rightarrow\left(Y, \tau_{2}\right)$ is fuzzy I-continuous and $\tau_{1}^{*}$ and $\tau_{2}^{*}$ be the associated fuzzy infi topology with $\tau_{1}$ and $\tau_{2}$ respectively. Suppose that $\mu \in \tau_{1}$, then $\mu \in \tau_{1}^{*}$. Now since $f$ is fuzzy continuous, $f^{-1}(\mu) \in \tau_{2}$, consequently $f^{-1}(\mu) \in \tau_{2}^{*}$. Thus $f$ is fuzzy I-continuous.

\subsection{Remark}

The converse of the above implication is not true, in general. It is shown in the following example. 


\subsection{Example}

Let $\lambda_{1}, \lambda_{2}$ and $\lambda_{3}$ be fuzzy subsets of $X=I$, defined as,

$$
\begin{gathered}
\lambda_{1}(x)= \begin{cases}0 & \text { if } 0 \leq x \leq \frac{1}{2} \\
2 x-1 & \text { if } \frac{1}{2} \leq x \leq 1 ;\end{cases} \\
\lambda_{2}(x)= \begin{cases}1 & \text { if } 0 \leq x \leq \frac{1}{4} \\
-4 x+2 & \text { if } \frac{1}{4} \leq x \leq \frac{1}{2} \\
0 & \text { if } \frac{1}{2} \leq x \leq 1\end{cases}
\end{gathered}
$$

and

$$
\lambda_{3}(x)= \begin{cases}1 & \text { if } 0 \leq x \leq \frac{1}{2} \\ -2 x+2 & \text { if } \frac{1}{2} \leq x \leq 1\end{cases}
$$

Let $\tau_{1}=\left\{0_{X}, \lambda_{1}, \lambda_{2}, \lambda_{1} \vee \lambda_{2}, 1_{X}\right\}$ be a fuzzy topology on $X$ and $\tau_{1}^{*}=$ $\left\{0_{X}, \lambda_{1}, \lambda_{2}, \lambda_{3}, \lambda_{1} \vee \lambda_{2}, \lambda_{1} \wedge \lambda_{3}, \lambda_{2} \wedge \lambda_{3}, 1_{X}\right\}$ be an associated fuzzy infi topology with $\tau_{1}$. Let $g: X \rightarrow X$ be a function defined by $g(x)=\frac{1}{2} x$.

Now

$$
\begin{aligned}
& \lambda_{1} \vee \lambda_{2}= \begin{cases}1 & \text { if } 0 \leq x \leq \frac{1}{4} \\
-4 x+2 & \text { if } \frac{1}{4} \leq x \leq \frac{1}{2} \\
2 x-1 & \text { if } \frac{1}{2} \leq x \leq 1\end{cases} \\
& \lambda_{1} \wedge \lambda_{3}= \begin{cases}0 & \text { if } 0 \leq x \leq \frac{1}{2} \\
2 x-1 & \text { if } \frac{1}{2} \leq x \leq \frac{3}{4} \\
-2 x+2 & \text { if } \frac{3}{4} \leq x \leq 1\end{cases}
\end{aligned}
$$

and

$$
\lambda_{2} \wedge \lambda_{3}= \begin{cases}1 & \text { if } 0 \leq x \leq \frac{1}{4} \\ -4 x+2 & \text { if } \frac{1}{4} \leq x \leq \frac{1}{2} \\ 0 & \text { if } \frac{1}{2} \leq x \leq 1\end{cases}
$$

so that, $g^{-1}\left(0_{X}\right)=0_{X}, g^{-1}\left(1_{X}\right)=1_{X}, g^{-1}\left(\lambda_{1}\right)=0_{X}, g^{-1}\left(\lambda_{2}\right)=\lambda_{3}, g^{-1}\left(\lambda_{1} \vee\right.$ $\left.\lambda_{2}\right)=\lambda_{3}, g^{-1}\left(\lambda_{1} \wedge \lambda_{3}\right)=0_{X}$, and $g^{-1}\left(\lambda_{2} \wedge \lambda_{3}\right)=\lambda_{3}$. Thus $g$ is fuzzy Icontinuous. But since $g^{-1}\left(\lambda_{1} \vee \lambda_{2}\right)=\lambda_{3}$ and $\lambda_{3} \notin \tau_{1}$, therefore $g$ is not a continuous function.

\subsection{Theorem}

The composition of two fuzzy I-continuous functions is fuzzy I-continuous.

Proof: The proof is obvious. 


\subsection{Corollary}

Suppose $\left(X, \tau_{1}\right),\left(Y, \tau_{2}\right),\left(Z, \tau_{3}\right)$ be fuzzy topological spaces and $\tau_{1}^{*}, \tau_{2}^{*}, \tau_{3}^{*}$ be the associated infi fuzzy topologies with $\tau_{1}, \tau_{2}, \tau_{3}$ respectively. If a fuzzy mapping $f:\left(X, \tau_{1}^{*}\right) \rightarrow\left(Y, \tau_{2}^{*}\right)$ is fuzzy I-continuous and $g:\left(Y, \tau_{2}\right) \rightarrow\left(Z, \tau_{3}\right)$ is fuzzy continuous, $g \circ f$ is fuzzy I-continuous.

\subsection{Definition}

Let $\left(X, \tau_{1}\right),\left(Y, \tau_{2}\right)$ be two fuzzy infi topological spaces and $f:\left(X, \tau_{1}\right) \rightarrow$ $\left(Y, \tau_{2}\right)$ be a function. Then $f$ is said to be fuzzy infi open if $f(\lambda)$ is fuzzy infi open in $Y$, for each fuzzy infi open set $\lambda$ in $X$. It is said to be fuzzy infi closed if $f(\lambda)$ is fuzzy infi closed in $Y$, for each fuzzy infi closed set $\lambda$ in $X$.

\subsection{Remark}

It is clear that a fuzzy open mapping (resp. fuzzy closed mapping) is fuzzy infi open (resp. fuzzy infi closed). The converse is not true, in general, as the following example will shows.

\subsection{Example}

Let $X=I$. Let us consider the fuzzy sets $\lambda, \mu$ and $\nu$ be fuzzy sets given by:

$$
\begin{aligned}
& \lambda(x)= \begin{cases}1 & \text { if } 0 \leq x \leq \frac{1}{3} ; \\
\frac{1}{3} & \text { if } \frac{1}{3} \leq x \leq 1 ;\end{cases} \\
& \mu(x)= \begin{cases}\frac{1}{2} & \text { if } 0 \leq x \leq \frac{1}{3} \\
\frac{1}{3} & \text { if } \frac{1}{3} \leq x \leq 1 ;\end{cases}
\end{aligned}
$$

and

$$
\nu(x)= \begin{cases}\frac{1}{3} & \text { for all } 0 \leq x \leq 1\end{cases}
$$

Let $\tau_{1}=\left\{0_{X}, \lambda, 1_{X}\right\}$ be any fuzzy topology defined on $X$. Then $\tau_{1}^{*}=\left\{0_{X}, \lambda, \mu, \nu, 1_{X}\right\}$ is an associated fuzzy infi topology with $\tau_{1}$ (since, $\lambda \wedge \mu=\lambda \wedge \nu=\mu \wedge \nu=\nu$.). We define $f$, a function from $X$ into $Y$ as $f(x)=\frac{(x+1)}{3}$. 
Then $f\left(0_{X}\right)=0_{X}, f\left(1_{X}\right)=1_{X}, f(\lambda)=f(\mu)=f(\nu)=\nu$. Thus we have, $f$ is fuzzy infi open, but since $f(\lambda)=\nu \notin \tau_{1}$, therefore $f$ is not fuzzy open.

\subsection{Theorem}

Let $f: X \rightarrow Y$ be a mapping from a fuzzy infi topological space $X$ into another fuzzy infi topological space $Y$. Then, $f$ is fuzzy infi open iff for each fuzzy point $\lambda_{x}$ of $X$ and each fuzzy infi open set $\lambda$ in $X$ containing $\lambda_{x}$, there exists a fuzzy infi open set $\mu$ in $Y$ containing $f\left(\lambda_{x}\right)$ such that $\left.\mu_{(} f(x)\right) \leq f(\lambda)$.

Proof: Necessary part is obvious.

To prove the sufficient part, let $\lambda$ be a fuzzy infi open set in $X$. Now by the given condition, for each fuzzy point $\lambda_{x}$ of $X$ such that $\lambda_{x} \in \lambda$, there exists a fuzzy infi open set $\mu$ of $Y$ containing $f\left(\lambda_{x}\right)$ such that $\mu_{(f(x))} \leq f(\lambda)$. Then, we have, $f(\lambda)=f\left(\bigcup_{\lambda_{x} \in \lambda} f\left(\lambda_{x}\right)=\bigcup_{\lambda_{x} \in \lambda} f\left(\lambda_{x}\right) \leq \bigcup_{\lambda_{x} \in \lambda} \mu_{(f(x))} \leq\right.$ $f(\lambda)$, that is, $f(\lambda)=\bigcup_{\lambda_{x} \in \lambda} \mu_{(f(x))}$ is a fuzzy infi open set in $Y$. Therefore $f$ is fuzzy infi open.

\subsection{Theorem}

Let $f: X \rightarrow Y$ be a mapping from a fuzzy infi topological space $X$ into another fuzzy infi topological space $Y$. Then $f$ is fuzzy infi closed iff $(f(\lambda))_{i c} \leq f\left(\lambda_{i c}\right)$, for each fuzzy set $\lambda$ in $X$.

Proof: It is obvious.

\subsection{Theorem}

The composition of two fuzzy infi open mapping is fuzzy infi open. Also, the composition of two fuzzy infi closed mapping is fuzzy infi closed.

Proof: It is obvious.

\section{Product Fuzzy Infi Topological Spaces}

In this particular section we establish the existence of product fuzzy infi topological space and we study some properties of fuzzy I-continuous function on product fuzzy infi topological space. 


\subsection{Lema}

The intersection of any collection of fuzzy infi topological spaces is a fuzzy infi topological space.

Proof: Let $\mathcal{B}=\{K: K$ is a fuzzy infi topology on $\mathrm{X}\}$ and $P=\cap\{K$ : $K \in \mathcal{B}$. To prove $P$ is a fuzzy infi topology.

Clearly $0_{X}, 1_{X} \in P$, since $0_{X}, 1_{X} \in K$, for each $K \in \mathcal{B}$.

Let us consider a finite family of fuzzy infi topology say, $\tau_{1}, \tau_{2}, \ldots ., \tau_{n} \in P$ and so $\tau_{1}, \tau_{2}, \ldots \ldots, \tau_{n} \in C$, and consequently belongs to $\bigwedge_{i=1}^{n} \tau_{i}$, for each $C \in \mathcal{B}$. So $\bigwedge_{i=1}^{n} \tau_{i} \in P$.

Hence, the intersection of any collection of fuzzy infi topology is again a fuzzy infi topology.

\subsection{Theorem}

Suppose $(X, \tau)$ be a fuzzy infi topological space and $f: X \rightarrow Y$ a fuzzy function. Then there is a weak fuzzy infi topology on $X$ for which $f$ is fuzzy I-continuous.

Proof: Suppose $\mathcal{B}=\left\{\tau^{*}: \tau^{*}\right.$ is a fuzzy infi topology on $X, f:\left(X, \tau^{*}\right) \rightarrow$ $(Y, \tau)$ a fuzzy I-continuous function $\}$. Clearly $I^{X} \in \mathcal{B}$ and so $\mathcal{B} \neq 0_{X}$. Then by lemma $4.1, \tau_{0}=\cap\left\{\tau^{*}: \tau^{*} \in \mathcal{B}\right\}$ is a fuzzy infi topology on $X$, which is a weak fuzzy infi topology on $X$ such that $f$ is a fuzzy I-continuous function. It is pertinent to mention here that, the weak fuzzy infi topology $\tau_{0}$ is the coarsest fuzzy infi topology on $X$ such that each element in $X^{*}$ (dual space) remains a continuous function.

\subsection{Corollary}

Suppose $(X, \tau)$ be a fuzzy infi topological space and $Y \subseteq X$. Then there is a weak fuzzy infi topology on $Y$, say $\tau^{*}$, such that the inclusion map $i:\left(Y, \tau^{*}\right) \rightarrow(X, \tau)$ is fuzzy I-continuous.

\subsection{Corollary}

Suppose $Y \subseteq X$ and $f:(X, \tau) \rightarrow\left(Z, \tau_{0}\right)$ are fuzzy I-continuous. Then $f \mid Y$ is fuzzy I-continuous if $Y$ is endowed with induced fuzzy infi topology.

Proof: Clearly, $f \mid Y=f \circ i$, where $i: Y \rightarrow X$ is the inclusion map and by theorem $4.2, Y$ is endowed with induced fuzzy infi topology. Then by 
Corollary 4.3, $i: Y \rightarrow X$ is fuzzy I-continuous. Again, by Theorem 3.16, the composition of two fuzzy I-continuous function is fuzzy I-continuous and hence $f \mid Y$ is fuzzy I-continuous.

\subsection{Theorem}

Consider a family $\left\{\left(X_{i}, \tau_{i}\right), i \in I\right\}$ of fuzzy infi topological spaces and $f_{i}$ : $X \rightarrow X_{i}$, a family of fuzzy functions. Then there is a weakest fuzzy infi topology on $X$ such that each $f_{i}$ is fuzzy I-continuous.

Proof: From the Theorem 4.2, for any $i \in I$ there is a weakest fuzzy infi topology $C_{i}$ on $X$ for which $f_{i}:\left(X, C_{i}\right) \rightarrow\left(X_{i}, \tau_{i}\right)$ is a fuzzy I-continuous function. Then, clearly, $C=\cup\left\{C_{i}: i \in I\right\}$ is the subbase for a fuzzy infi topology on $X$ making each $f_{i}$ fuzzy I-continuous. Moreover, it can be easily verified that this is the smallest fuzzy infi topology with this property.

\subsection{Definition}

The weakest fuzzy infi topology generated on $X$ by the family $\left\{\left(X_{j}, \tau_{j}\right), j \in\right.$ $J\}$ of fuzzy infi topological spaces and a family of fuzzy functions $f_{j}: X \rightarrow$ $X_{j}$ is called the initial fuzzy infi topological space that makes each $f_{j}$ fuzzy I-continuous.

\subsection{Corollary}

For any set $Y$, a fuzzy infi topological space $(X, \tau)$ and a function $f: X \rightarrow$ $Y$ it can be easily verified that $D=\left\{\delta \in I^{Y}: f^{-1}(\delta) \in \tau\right\}$ is the finest fuzzy infi topology on $Y$ making $f$ a fuzzy I-continuous function. More generally, consider a family of fuzzy infi topological spaces $\left\{\left(X_{j}, \tau_{j}\right), j \in J\right\}$ and for each $j \in J$, a function $f_{j}: X_{j} \rightarrow Y$, then the intersection $\cap_{j} D_{j}$ is the finest fuzzy infi topology on $Y$ making all $f_{j}$ fuzzy I-continuous functions, where for each $j \in J, D_{j}$ is the finest fuzzy infi topology on $Y$ for which $f_{j}:\left(X_{j}, \tau_{j}\right) \rightarrow\left(Y, D_{j}\right)$ is fuzzy I-continuous. This topology $\cap_{j} D_{j}$ on $Y$ is called the final fuzzy infi topology.

\subsection{Theorem}

Consider a family $f_{j}: X \rightarrow X j, j \in J$, of functions, where $\left(X j, \tau_{j}\right)$ are fuzzy infi topological spaces and $X$ is a given set equipped with the initial fuzzy infi topology $\tau$. Then, a function $f:\left(Y, \tau^{*}\right) \rightarrow(X, \tau)$ is fuzzy I-continuous iff $f_{j} \circ f$ is a fuzzy I-continuous function for all $j \in J$. 
Proof: If $f$ is a fuzzy I-continuous function, then from Theorem 3.16, for each $j \in J, f_{j} \circ f$ is a fuzzy I-continuous function. Conversely, let if possible, each $f_{j} \circ f$ be a fuzzy I-continuous function for each $j \in J$, with $f$ is not a fuzzy I-continuous function, which means that there is $\lambda \in \tau$ such that $f^{-1}(\lambda) \in \tau^{*}$. Then one of the following two statements holds:

(i) for any particular $i \in J$, there exists $\lambda_{i} \in \tau_{i}$ such that $\lambda=f i^{-1}\left(\lambda_{i}\right)$,

(ii) for all $j \in J$ and $\lambda_{j} \in \tau_{j}, \lambda \neq f_{j}^{-1}\left(\lambda_{j}\right)$.

In case (i), we have

$$
f^{-1}(\lambda)=f^{-1}\left(f_{i}^{-1}\left(\lambda_{i}\right)\right)=\left(f_{i} \circ f\right)^{-1}\left(\lambda_{i}\right) .
$$

Therefore $\left(f_{i} \circ f\right)^{-1} \in \tau^{*}$, which is a contradiction to the fact that $f_{i} \circ f$ is fuzzy I-continuous.

In case $(i i)$, since $f^{-1}\left(0_{X}\right)=0_{Y}$ and $f^{-1}\left(1_{X}\right)=1_{Y}$, so $\lambda \neq 0_{X}, 1_{X}$. Then $\tau-\{\lambda\}$ is a fuzzy infi topology on $X$. But for each $j \in J, f_{j}:(X, \tau-\{\lambda\}) \rightarrow$ $\left(X_{j}, \tau_{j}\right)$ is fuzzy I-continuous, which is a contradiction with the choice of $\tau$, as $\tau$ is the weakest fuzzy infi topology on $X$.

Thus $f$ is fuzzy I-continuous.

As a consequence of Theorem 4.5 we now present a product fuzzy infi topology for an arbitrary family $\left\{\left(X_{j}, \tau_{j}\right): j \in J\right\}$ of fuzzy infi topological spaces. The product fuzzy infi topology on $X=\prod_{j \in J} X_{j}$ is the weakest fuzzy infi topology on $X$, such that for each $k \in J$, the projection $\pi_{k}$ : $\prod_{j \in J} X_{j} \rightarrow X_{k}$ is a fuzzy I-continuous function.

\subsection{Corollary}

For any family $\left\{\left(X_{j}, \tau_{j}\right): j \in J\right\}$ of fuzzy infi topological spaces, a fuzzy product fuzzy infi topology on $X=\prod_{j \in J} X_{j}$ exists.

Proof: In Theorem 4.5, if we replace $f_{j}$ by $\pi_{j}$, then we get the required theorem.

\subsection{Corollary}

Suppose the family $\left(X_{j}, \tau_{j}\right)$ consists of fuzzy infi topological spaces and $X=\prod_{j \in J} X_{j}$ is equipped with the product fuzzy infi topology generated by projection maps $\left\{\pi_{j}: X \rightarrow X_{j}, j \in J\right\}$. Then $f:\left(Y, \tau^{*}\right) \rightarrow(X, \tau)$ is a fuzzy I-continuous function iff $\pi_{j} \circ f$ is a fuzzy I-continuous function for all $j \in J$.

Proof: The proof follows immediately from Theorem 4.8 . 


\subsection{Corollary}

Suppose $f:(X, \tau) \rightarrow\left(Y, \tau^{*}\right)$ and $g:(X, \tau) \rightarrow\left(Z, \tau_{0}\right)$ are two fuzzy Icontinuous functions. Then the function $f \times g:(X, \tau) \rightarrow\left(Y \times Z, \tau^{*} \times \tau_{0}\right)$ defined by $(f \times g)(x)=(f(x), g(x))$ is fuzzy I-continuous.

Proof: Consider $\pi_{1}$ and $\pi_{2}$ such that $\left(\pi_{1} \circ(f \times g)\right)(x)=\pi_{1}(f(x), g(x))=$ $f(x)$ and $\left(\pi_{2} \circ(f \times g)\right)(x)=\pi_{2}(f(x), g(x))=g(x)$.

Clearly, $\left(\pi_{1} \circ(f \times g)\right)$ and $\left(\pi_{2} \circ(f \times g)\right)$ are fuzzy I-continuous functions. Hence by Corollary $4.10, f \times g$ is fuzzy I-continuous.

\section{Conclusion}

In [6], we introduced and discussed the concept of infi topological spaces. In [13] Saha and Bhattacharya introduced the concept of induced fuzzy infi topological space. They showed the existence of this space with the help of NLSC functions. But they didnt go further. Fuzzy topological space is such a concept which created a great impact in applied math and in different dimensions of physics also like quantum physics, string theory, $\varepsilon^{\infty}$-theory . Now since fuzzy infi topological space is a generalization of fuzzy topological space, we contemplate this space also, is very much convenient in those fields. In this paper we went on to study some basic properties of fuzzy infi topological space like fuzzy infi interior, fuzzy infi closure, fuzzy I-continuous functions, composition of fuzzy I-continuous functions, fuzzy infi open mappings, fuzzy infi closed mappings etc and also established some theorems related to those concepts. Further we established the existence of product fuzzy infi topological space and studied some properties of fuzzy I-continuous functions on product fuzzy infi topological space. There is a scope to study the notion of fuzzy connectedness, fuzzy hyper connectedness, fuzzy locally connectedness, fuzzy extremely disconnectedness, fuzzy sub maximal spaces etc in a given fuzzy infi topological space.

\section{References}

[1] M. Alimohammady and M. Roohi, "Compactness in fuzzy minimal spaces", Chaos, solitons \& fractals, vol. 28, no. 4, pp. 906-912, 2006, doi: 10.1016/j.chaos.2005.08.043 
[2] M. Alimohammady and M. Roohi, 2006, "Fuzzy minimal structure and fuzzy minimal vector spaces", Chaos, solitons \& fractals, vol. 27, no. 3, pp. 599-605, 2006, doi: 10.1016/j.chaos.2005.04.049

[3] M. Alimohammady, E. Ekici, S. Jafari, and M. Roohi, "Fuzzy minimal separation axioms", Journal of nonlinear sciences and applications, vol. 03, no. 03, pp. 157-163, 2010, doi: 10.22436/ jnsa.003.03.01

[4] G. Balasubramanian and P. Sundaram, "On some generalizations of fuzzy continuous functions", Fuzzy sets and systems, vol. 86, no. 1, pp. 93-100, 1997, doi: 10.1016/ 0165-0114(95)00371-1

[5] C. L. Chang, "Fuzzy topological spaces", Journal of mathematical analysis and applications, vol. 24, no. 1, pp. 182-190, 1968, doi: 10.1016/ 0022-247X(68)90057-7

[6] B. Das, A. K. Saha and B. Bhattacharya, "On Infi-topological spaces", Journal of fuzzy mathematics, vol. 25, no. 2, pp. 437-448, 2017.

[7] S. Dang, A. Behera, and S. Nanda, "Some results on fuzzy supratopological spaces", Fuzzy sets and systems, vol. 62, no. 3, pp. 333-339, 1994, doi: 10.1016/ 0165-0114(94)90117-1

[8] R. Lowen, "Fuzzy topological spaces and fuzzy compactness", Journal of mathematical analysis and applications, vol. 56, no. 3, pp. 621-633, 1976, doi: 10.1016/ 0022-247X(76)90029-9

[9] H. Maki, J. Umehara, and T. Noiri, "Every topological space is pre $\mathrm{T}_{1 / 2}$ ", Memoirs of the Faculty of Science Kochi University Series A Mathematics, vol. 17, pp. 33-42, 1996.

[10] A. S. Masshour, A. A. Allam, F. S. Mahmud, and F. H. Khedr, "On supratopological spaces", Indian journal of pure and applied mathematics, vol. 14, no. 4, pp. 502-510, 1983. [On line]. Available: https:/ / bit.ly/ 3dUcp63

[11] M. E. A. E. Monsef, and A. E. Ramadan, "On fuzzy supra topological spaces", Indian journal of pure and applied mathematics, vol. 18, no. 4, pp. 322-329, 1987. [On line]. Available: https:/ / bit.ly/ 3b12z0p

[12] G. Palani Chetty, "Generalized fuzzy topology", Italian journal of pure and applied mathematics, no. 24, pp. 91-96, 2008.

[13] A. K. Saha, and D. Bhattacharya, "Normal induced fuzzy topological spaces", Italian journal of pure and applied mathematics, no. 34, pp. 45-56, 2015. [On line]. Available: https:// bit.ly/ 3r5JAaA

[14] A. P. Šostak, "On a fuzzy topological structure", Rendiconti del Circolo Matematico di Palermo, Serie II, Supplemento, no. 11, pp. 89-103, 1985. [On line]. Available: https:/ / bit.ly/ 37T4IJL 
[15] B. C. Tripathy and G. C. Ray, "On $\delta$-continuity in mixed fuzzy topological spaces", Boletim da Sociedade Paranaense de Matemática, vol. 32, no. 2, pp. 175, 2014, doi: 10.5269/ bspm.v32i2.20254

[16] B. C. Tripathy and G. C. Ray, "Mixed fuzzy ideal topological spaces", Applied mathematics and computation, vol. 220, pp. 602-607, 2013, doi: 10.1016/j.amc.2013.05.072

[17] B. C. Tripathy and G. C. Ray, "Fuzzy $\delta$-almost continuous and fuzzy $\delta *$-continuous functions in mixed fuzzy ideal topological spaces", Proyecciones (Antofagasta), vol. 39, no. 2, pp. 435-449, 2020, doi: 10.22199/ issn.0717-6279-2020-02-0027

[18] L. A. Zadeh, "Fuzzy sets", Information and control, vol. 8, no. 3, pp. 338353, 1965, doi: 10.1016/ S0019-9958(65)90241-X 